\section{Mucocele of the appendix in a 77-year-old man with calcinosis, Raynaud's phenomenon, esophageal dysfunction, sclerodactyly and telangiectasias syndrome}

Hanen Loukil, Faten Frikha, Mouna Snoussi, Zouhir Bahloul

Department of Internal Medicine, Hedi Chaker Hospital, Sfax, Tunisia

\section{Abstract}

Mucocele is an uncommon pathology of the vermiform appendix estimated to be seen in $0.2-0.3 \%$. The term mucocele means dilation of the appendix due to mucus, caused either by a benign or a malignant process. Herein, we report the case of a 77-year-old man with Calcinosis, Raynaud's phenomenon, Esophageal dysfunction, Sclerodactyly and Telangiectasias syndrome, a limited form of Scleroderma, who had presented an abdominal cyclical pain and in which abdominopelvic computed tomography scan concluded to the diagnosis of appendiceal mucocele. Surgery and histopathology confirmed the diagnosis of mucinous cystadenoma. This association appendiceal mucocele and scleoderma has not been previously reported. The clinical and radiological features of this unusual complication are reviewed.

\section{Introduction}

The term mucocele of the appendix or appendiceal mucocele (AM) describes a progressive dilatation of the appendix as a result of intra-luminal accumulation of the mucoid substance. It is a rare entity that has been reported in $0.1-0.4 \%$ of all appendectomy specimens ${ }^{1,2}$ and can present with a variety of clinical symptoms or occur as an incidental surgical finding. The most common clinical forms of presentation are clinical symptoms of pain or discomfort at the height of the right lower quadrant indicative of acute appendicitis, or as an abdominal mass found in the right iliac fossa. ${ }^{3}$ The major pathological mechanisms are thought to be responsible for the formation of AM are elevated appendiceal pressures as sequelae of luminal obstruction caused by prior inflammation, mucosal hyperplasia, or appendiceal lesions (fecaliths, endometriosis, diverticulae, polyps) and tumors of the appendix (carcinoid, cystadenoma, cystadenocarci- noma). ${ }^{4}$ While scleroderma [systemic sclerosis (SSc)] can lead to cutaneous mucinos; an association with AM of the appendix has not been previously reported. We report a case of $\mathrm{AM}$ in a patient with Calcinosis, Raynaud's phenomenon, Esophageal dysfunction, Sclerodactyly and Telangiectasias (CREST) syndrome, a limited variant of Scleroderma.

\section{Case Report}

A 77-year-old man, who is followed in our department of Internal Medicine since 2004 for limited cutaneous scleroderma (CREST syndrome), was admitted for recent abdominal pain. In his past medical history, the patient had developed Reynaud's phenomenon involving upper limbs, dysphagia, and sclerodactyly along with digital infarction and multiple telangiectasias of hands and face (Figures 1 and 2). Autoimmune profile revealed positive anti-nuclear antibodies (titer 1/800); positive anti-centromere antibody, negative anti-scl70, anti-double-stranded DNA, anti-cardiolipin and lupus anticoagulant antibodies. A radiograph of both hands showed terminal tuft resorption of most digits. He had been diagnosed as having CREST syndrome. He had received low dose of steroid (10 $\mathrm{mg} /$ day) and vasodilator drugs with a good clinical course during 10 years.

Concerning abdominal pain, it was a cyclical pain that had increased in intensity over the past 7 days and now radiated somewhat more superiorly and towards the right flank. The patient denied any fever, chills, nausea, vomiting or diarrhea. His last bowel movement had been the day before presentation and was described as normal. His physical examination revealed moderate tenderness in the right lower quadrant at McBurney's point and some voluntary guarding. No abdominal masse was noted. The rectal examination was normal. Laboratory studies included a normal urine analysis and complete blood count (hemoglobin $12 \mathrm{~g} / \mathrm{dL}$, white blood cells account at 8200 $\mathrm{mm}^{3}$ with $62 \%$ polymorph nuclear leukocytes). Ultrasound showed liquid mass simulating a hydatid cyst.

An abdominopelvic computed tomography (CT) scan, with oral and intravenous contrast enhancement, demonstrated a $6 \mathrm{~cm}$ diameter retrecoecale cystic mass, which goes up in liver and extended to a height of $8 \mathrm{~cm}$. A small round calcified density was noted along the wall (Figure 3 ). This mass was seen also protruding into the cecum from the appendicle orifice. The diagnosis of appendiceal mucocele was suspected. The patient was referred to the department of surgery and an open surgery was performed in emergency. At the time of surgery, a cystic mass of the appendix $(8 \times 4 \times 3$
Correspondence: Faten Frikha, Department of Internal Medicine, Hedi Chaker Hospital, 3029 Sfax, Tunisia.

E-mail: fetenfrikha@yahoo.fr

Key words: Mucocele; Appendix; Scleroderma; CREST syndrome.

Contributions: the authors contributed equally.

Conflict of interest: the authors declare no potential conflict of interest.

Received for publication: 7 March 2016. Accepted for publication: 29 August 2016.

This work is licensed under a Creative Commons Attribution NonCommercial 4.0 License (CC BYNC 4.0).

(C) Copyright H.Loukil et al., 2016

Licensee PAGEPress, Italy

Gastroenterology Insights 2016; 7:6169

doi:10.4081/gi.2016.6169

cm), with inflamed walls, but without perforation, was discovered in the right iliac fossa. No discharge was found in the peritoneal cavity.

The exploration of the meso-ascending colon detects multiple lymphadenopathies along the ileo bicoeco trunk and the appendicular artery of the upper barter mesenteries. The lymph nodes were increased in size with a suspicious appearance. A hemicolectomy was performed. Histopathology diagnosis was consistent with mucinous cystadenoma. There were no complications in the postoperative period and the patient was released from the hospital on the fifth day.

\section{Discussion}

$\mathrm{AM}$ is not a specific diagnosis, but rather, is a descriptive term for dilatation of the lumen of the vermiform appendix by an abnormal accumulation of mucous. It is a very uncommon disease estimated to be seen in $0.1-0.4 \%$ of all appendectomies performed and $8-10 \%$ of all appendiceal tumors. ${ }^{3}$ Since 1842 , when Rokitansky presented the mucocele as a pathological entity, four few series have been published on this type of tumor. ${ }^{5}$ Of these, one of the broadest in the medical literature was published in 2003 at the Mayo Clinic with 132 patients. ${ }^{6}$ In fact, the term mucocele referring to an enlarged appendix and by mucoid content including both benign and malignant processes.

Elbe $^{7}$ has stated that a number of factors are essential for the development of a mucocele: i) a gradual obliteration of the lumen of the 
appendix at one or more points; ii) the absence of grossinfection; iii) a mucosa which is capable of active secretion of mucus, or a state of affairs in which absorption is less than secretion; iv) and distension. The transformation of mucin into pseudomucin must also be included. ${ }^{7}$ In the majority of cases the obliteration of the lumen is the result of chronic appendicitis. This obliteration is common at the base of the appendix. A similar result may also be a sequel of acute appendicitis.

Microscopic mucin deposition within the dermis has been described in patients with connective tissue disorders including Systemic Lupus Erythematous (SLE) and scleroderma. ${ }^{8,9}$ In the literature, this entity is extremely rare in association with connective. A MEDLINE

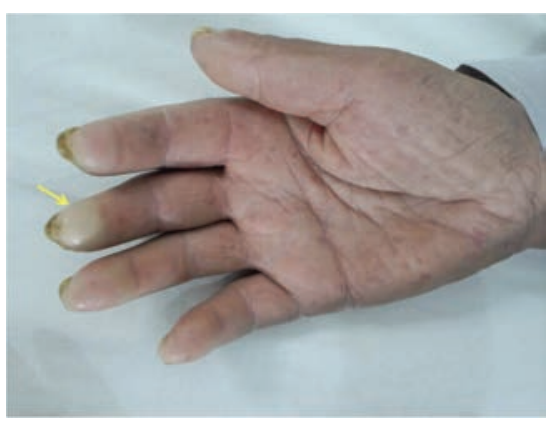

Figure 1. Raynaud's phenomenon of fingers with telangiectasias of the right hand.

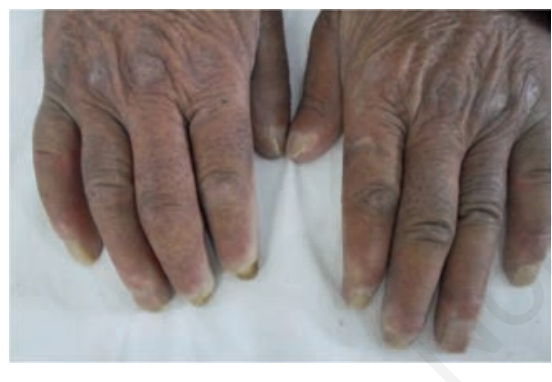

Figure 2. Sclerodactyly of both hands.

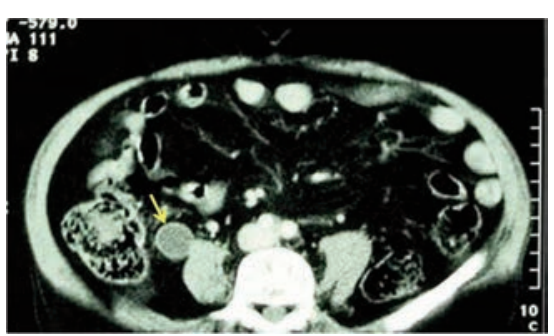

Figure 3. Abdominopelvic computed tomography scan, with contrast enhancement, demonstrated a $6 \mathrm{~cm}$ diameter retrecoecale cystic mass. A small round calcify density was noted along the wall (arrow) consistent with the diagnosis. search of published items from 1985 to 2014 inclusive by using the text words mucinous cystadenoma or mucinous cystadenocarcinoma and systemic sclerosis or scleroderma or CREST syndrome failed to reveal any reported cases. There was only one case report of mucinous cystadenoma of the appendix in a patient with SLE. ${ }^{10}$ We also found one case of a large mucocele of the esophagus in a 26-year-old woman who had undergone esophageal bypass surgery and gastric interposition because of involvement by scleroderma. ${ }^{11}$ Although the hypothesis that the abnormal mucin deposition in the cutaneous tissues of some SSc patients may have played a role in the development of mucinous cystadenoma in our patient, we thought that this lesion occurred coincidentally in this case. The average age at diagnosis is 50 years and, although early reports suggested that AM occurred more frequently in women than men, this predominance has been challenged in more recent studies. ${ }^{12}$ The patients are often asymptomatic. The most common presentation of symptomatic AM patients is acute or chronic right lower quadrant abdominal pain. ${ }^{13}$ Nausea and vomiting, as well as altered bowel habits (diarrhea, constipation) are often reported. An intra-abdominal mass is palpated by the examining physician in half of cases and is also occasionally palpated by the patient. ${ }^{14}$ Preoperative diagnosis of appendicular mucocele is very important for the selection of an adequate surgical method to prevent peritoneal dissemination, to prevent intraoperative and postoperative complications, and repeated surgery. ${ }^{15}$ Laboratory studies have a limited role in the diagnosis of AM and leukocytosis is generally absent. A complete blood count and urinalysis are used to help in excluding other pathologies. Ultrasound (US), CT, and colonoscopy are used for diagnosis. US is the first-line diagnosis modality for patients with acute abdominal pain or mass. Furthermore, US can be used to differentiate between mucocele and acute appendicitis. Therefore it is quite useful in diagnosing AM and offers the added advantage of allowing a concomitant evaluation of any other masses.

By colonoscopy, an elevation of the appendiceal orifice is seen and a yellowish mucous discharge would be visible from this orifice. Furthermore, synchronous and metachronous tumors of colon can be identified. ${ }^{16}$

CT is regarded as the most accurate method of diagnosis. It usually demonstrates a cystic, well-encapsulated mass occasionally with calcifications, as in the present case, in the area of the appendix impinging on the cecal wall but without evidence of inflammation. CT may also demonstrate the existence of concomitant pathology such as the presence of ascites, an important finding as it is indicative of pseudomyxoma peritonei. ${ }^{14,17}$ In our patient US did not provide the correct information; we thought that this was a hydatid cyst so we performed CT scan.

Magnetic resonance imaging has been reported useful for the evaluation of $\mathrm{AM}$ and also has the advantage of demonstrating any concomitant pathology. ${ }^{18}$ One of the cardinal principles of surgical treatment of this disease is that intact mucoceles do not pose a threat for the patient. If it is perforated and the filling turns up in the peritoneal cavity, there is a high probability that pseudomyxoma peritonei; the worst complication of AM. So the preoperative diagnosis that distinguishes appendiceal mucocele from acute appendicitis is essential for the best choice of surgical approach (open $v s$ laproscopic) to prevent peritoneal dissemination of mucin producing epithelial cells and to perform the appropriate surgery. ${ }^{15}$ Some surgeons think that open surgery should be favored against laparoscopy. The open method allows a carefully surgery so the cyst is not ruptured and the filling is not scattered into the peritoneal cavity. Also with an open surgery compared to the laparoscopic method, it is possible to the surgeon to more thoroughly explore the remainder of the abdomen: to have a fuller inspection, palpation, and direct inspection of the spots in the abdomen. But if the surgery was launched using a laparoscopic method and it appears that there is an appendiceal mucocele, it must be converted into open surgery. Conversion of a laparoscopy to a laparotomy for excision of a mucocele aids in managing this disease process by seeing that an intact mucocele is a benign process. An algorithm for the selection of the type of surgery has been provided by Dhage-Ivatury and Sugarbaker. This envisages several factors: i) whether or not a mucocele is perforated; ii) whether the base of the appendix (margins of resection) is involved in the process; and iii) whether there are positive lymph nodes of mesoappendix and ileocolic. As a result patients may require different operations: appendectomy to the right colectomy, including cytoreductive surgery, heated intraoperative intraperitoneal chemotherapy, early postoperative intraperitoneal chemotherapy.

In our patient the mucocele was not perforated (no discharge into the peritoneum cavity) but the regional lymph nodes were negative. Therefore, a right colectomy was performed, which is an adequate surgery in such a case. Follow-up of all patients is justified, because of the risk of recurrence in the form of pseudomyxoma peritonei or colorectal neoplasms.

\section{Conclusions}

In conclusion mucocele of the appendix is rare entity, manifested usually by nonspecific 
clinical signs. Ultrasound and CT are helpful in the preoperative diagnosis. The treatment of choice is appendectomy, although in mucinous cystoadenocarcinoma right hemicolectomy is needed. In our knowledge, this is the first case report of a mucocele of the appendix in a patient with Scleroderma.

\section{References}

1. Higa E, Rosal J, Pizzimbonoo C, Wise L. Mucosal hyperplasia, mucinous cystadenoma and mucinous cystadenocarcinoma of the appendix. Cancer 1973;32:1525-41.

2. Loizon P, Filali K, Lapeyrie H, Chapuis H. Appendiceal mucoceles. Apropos of 2 cases. J Chir (Paris) 1989;126:703-5.

3. García Lozano A, Vázquez Tarrago A, Castro García C, et al. Mucocele of the appendix: presentation of 31 cases. Cir Esp 2010;87:108-12.

4. Scotté $M$, Laquerrière $A$, Riff $Y$, et al. Appendiceal mucoceles: pathophysiology and therapeutic indications. J Chir (Paris)
1994;131:303-12.

5. Rokitansky CF. A manual of pathological anatomy. Vol. 2. Philadelphia: Blancard and Lea; 1842. pp 1855-89. English translation of the Vienna edition.

6. Stocchi L, Bruce G, Dirk R, Jeff R. Surgical treatment of appendiceal mucocele. Arch Surg 2003;138:585-90.

7. Elbe T. Mucocele of the vermiform appendix. Int Clinics 32nd Ser. II. 1922. p 303.

8. Alikhan M, Cumming OW, Rex D. Subtotal colectomy in a patient associated with collangeous colitis with colonic carcinoma and systemic lupus erythematosus. Am J Gastroenterol 1997;92:1213-5.

9. Gemke GR, Bilzens EP Senina VA. Bleeding polyp of the transverse colon in a patient with systemic lupus erythematosus. Klin Med (Mosk) 1983;61:111-2.

10. Wirtzfeld DA, Price LM, Duggan MA, et al. Mucinous cystadenoma of the appendix in a patient with systemic lupus erythematosus. Can J Gastroenterol 1998;12:573-6.

11. Van Gelderen WF. Mucocele of the surgically isolated esophagus. Abdom Imaging 1993;18:13-4.
12. Souie-Mhiri M, Tlili-Graies K, Ben Cherifa $\mathrm{L}$, et al. Mucocele of the appendix. Review of 10 cases. J Radiol 2001;82:463-8.

13. Hamilton DL, Stormont JM. The volcano sign of appendiceal mucocele. Gastrointest Endosc 1989;35:453-6.

14. Coulier B, Pestieau S, Hamels J, Lefebvre Y. US and CT diagnosis of complete cecocolic intussusception caused by an appendiceal mucocele. Eur Radiol 2002;12:324-8.

15. Dhage-Ivatury S, Sugarbaker PH. Update on the surgical approach to mucocele of the appendix. J Am Coll Surg 2006;202: 680-4.

16. Zanati SA, Martin JA, Baker JP, et al. Colonoscopic diagnosis of mucocele of the appendix. Gastrointest Endosc 2005;62: 452-6.

17. Ruiz-Tovar J, Teruel DG, Gastineires VM, et al. Mucocele of the appendix. World $\mathrm{J}$ Surg 2007;31:542-8.

18. Jaluvka V, Albig M, Hamm B. Magnetic resonance tomography in the differential diagnosis of ovarian tumor and mucocele of the appendix. Dtsch Med Wochenschr 1989;114:1245-7. 